Sara Rosa de Sousa AndRade ${ }^{1}$

Fernanda Graziele da Silva Azevedo Nora ${ }^{2}$

Ivan Silveira de AvelaR ${ }^{1}$

VIVIANE SOARES ${ }^{1}$

Waldemar NAVEs do Amaral ${ }^{1}$

Marcus Fraga VierRA ${ }^{3}$

Artigo Original

Palavras-chave

Gestantes

Marcha

Biomecânica

Keywords

Pregnant women

Gait

Biomechanics

\title{
Avaliação da inicialização da marcha na gestante de baixo risco nos três trimestres gestacionais
}

\author{
Evaluation of gait initiation in low risk pregnant women in the \\ three trimesters
}

Resumo

OBJETIVOS: Descrever o processo de inicialização da marcha de gestantes e comparar o comportamento do centro de pressão nos três trimestres gestacionais. MÉTODOS: Foram avaliadas 57 gestantes de baixo risco, com idades de 18 a 35 anos, em três trimestres gestacionais, selecionadas por conveniência. Foram dividas em três grupos por idade gestacional $-1^{\circ}$ trimestre (4-12 semanas), $2^{\circ}$ trimestre (13-28 semanas) e $3^{\circ}$ trimestre (29-42 semanas) - , sendo 19 gestantes para cada trimestre. Cada gestante foi posicionada em posição ortostática, com um pé em cada plataforma de força AMTI, ficando assim até ouvir um sinal sonoro para iniciar a marcha em um percurso de quatro metros. Os dados obtidos foram submetidos à análise estatística com o programa SPSS. Para a comparação dos grupos utilizou-se os teste de Kolmogorov Smirnov, teste de Tukey e o coeficiente de correlação de Spearman. Em todas as análises considerou-se o nível de significância de 5\%. RESULTADOS: Foram encontradas diferenças significativas quando comparados os grupos $1^{\circ}$ trimestre (GPT) e $3^{\circ}$ trimestre (GTT), para as variáveis amplitude de oscilação médio lateral GPT $(0,4 \mathrm{~cm})$ e GTT $(0,2 \mathrm{~cm})$ e velocidade de deslocamento médio-lateral - GPT $(0,9 \mathrm{~cm} / \mathrm{s})$ e GTT $10,4 \mathrm{~cm} / \mathrm{s})$. Observou-se uma diminuição gradativa nas variáveis de amplitude de oscilação anteroposterior e médiolateral, bem como, na velocidade de deslocamento do primeiro trimestre na plataforma 1 em relação à plataforma 2. Houve diferenças significativas nas variáveis de amplitude de oscilação médio-lateral e velocidade de deslocamento médio-lateral comparando GPT e GTT. CONCLUSÃO: As variáveis analisadas apresentaram pequenas diferenças e não constituem risco eminente para a estabilidade dinâmica da gestante.

\section{Abstract}

PURPOSES: To describe the process of gait initiation of pregnant women and to compare the behavior of the pressure center in the three trimesters of pregnancy. METHODS: Fifty-seven low-risk pregnant women were evaluated, aged 18 to 35 years, selected for convenience location during the three trimesters of pregnancy. The women were divided into three groups of 19 subjects each, according to gestational age - $1^{\text {st }}$ quarter (4-12 weeks), $2^{\text {nd }}$ quarter (1 3-28 weeks), and $3^{\text {rd }}$ quarter (29-42 weeks, ). Each patient was positioned standing up with one foot on each AMTI force platform until she heard a beep indicating that she should start walking a distance of four meter. Data were analyzed using the SPSS software. The Kolmogorov Smirnov test, Tukey's test and Spearman correlation coefficient were used for group comparisons, with 5\% significance level in all tests. RESULTS: Significant differences were found between the $1^{\text {st }}$ quarter (GFT) and $3^{\text {rd }}$ quarter (GT) groups regarding mediolateral oscillation amplitude (GFT: $0.4 \mathrm{~cm}$ and GTT: $0.2 \mathrm{~cm}$ ) and mediolateral displacement rate (GFT: $0.9 \mathrm{~cm} / \mathrm{s}$ and GT: $0.4 \mathrm{~cm} / \mathrm{s}$ ). There was a gradual decrease in anteroposterior and mediolateral oscillation rate, and in the speed of displacement from platform 1 to platform 2 in GFT. There was a significant difference in oscillation amplitude and mediolateral displacement speed between GFT and GTT. CONCLUSION: The variables analyzed showed minor differences and do not constitute an imminent risk for the stability dynamics of pregnant woman.

Correspondência

Sara Rosa de Sousa Andrade Laboratório de Bioengenharia e Biomecânica da Faculdade de Educacãōo Física da Universidade Federal de Goiás Campus Samambaia, Caixa Postal 131 CEP: $74001-970$

Goiânia (GO), Brasil

Recebido

$16 / 02 / 2012$

Aceito com modificacōes 02/08/2012
Trabalho realizado no Laboratório de Biomecânica e Bioengenharia da Universidade Federal da Goiás - UFG - Goiânia (GO), Brasil. 'Programa de Pós-graduação em Ciências da Saúde da Universidade Federal de Goiás - UFG - Goiânia (GO), Brasil. 2Programa de Pós-graduação em Fisioterapia da Universidade Federal de São Carlos - UFSCar - São Carlos (SP), Brasil. 3Programa de Pós-graduação em Engenharia Elétrica e de Computação da Universidade Federal de Goiás - UFG - Goiânia (GO), Brasil. 
Introdução

A gestação é um processo fisiológico no qual ocorre uma sequência de adaptações no corpo da mulher, a partir, do momento da fertilização ${ }^{1}$. A gravidez promove no organismo inúmeras transformações como a frouxidão ligamentar decorrente de ação hormonal e, também, modificações estruturais desde a coluna vertebral até a dinâmica da marcha. Outros fatores, como o aumento do peso, o crescimento do útero e das mamas contribuem para modificar as forças gravitacionais atuantes na gestação $0^{2,3}$.

Diversos estudos investigaram o controle postural no estado estacionário e no processo dinâmico da marcha de mulheres grávidas. Porém, a inicialização da marcha é bastante complexa, pois, o corpo sai de uma posição de "conforto" para iniciar o movimento, que é chamado primeiro passo. Este início pode ser afetado pelo aumento do peso que reduz a velocidade da marcha e induz o aparecimento de dor pélvica que causa desconforto para a grávida ${ }^{4,5}$.

A análise da inicialização da marcha é uma situação particularmente interessante, pois permite melhor compreensão dos mecanismos centrais e periféricos envolvidos neste processo. Como modelo nas interações entre o equilíbrio, postura e movimento voluntário, a inicialização da marcha analisa as necessidades posturais e dinâmicas que têm de ser satisfeitas antes de caminhar e, também, permite identificar adaptações no comando motor realizado pelo sistema nervoso central (SNC), verificando a ocorrência de algum déficit ${ }^{6}$.

O método utilizado para o estudo das alterações posturais estáticas e dinâmicas que ocorrem é comumente chamado de análise cinética, e esta analisa inúmeras variáveis tais como força de reação do solo, centro de pressão (COP), amplitude e velocidade do movimento. Quando há alteração nos fatores biomecânicos, associados à gestação, como o COP, padrão de marcha e ganho de peso, ela pode contribuir para ocorrências de disfunções musculoesqueléticas nos membros inferiores, principalmente, tensão e dor muscular, câimbras e fadiga na região do tronco e nos membros inferiores além de deixar a gestante mais susceptível a queda ${ }^{4}$.

Alguns estudos já mostraram resultados da análise da marcha sem a fragmentação das fases, o que oferece uma visão geral das alterações que ocorrem durante este processo. Em um estudo que avaliou a força de reação do solo, no último trimestre, no ciclo da marcha de mulheres grávidas com e sem relatos de quedas, não foi encontrada diferença entre os grupos estudados. No último trimestre há aumento do risco de quedas para as gestantes, visto que, neste período há um aumento de peso, maior retenção de líquido e um aumento da frouxidão ligamentar ${ }^{7}$. Com isso, o objetivo deste estudo foi descrever o processo de inicialização da marcha de gestantes e comparar o comportamento nos três trimestres gestacionais.

\section{Métodos}

Trata-se de um estudo descritivo transversal. A pesquisa incluiu gestantes atendidas no Serviço de Ginecologia de uma clínica na cidade de Goiânia (GO). A clínica cedeu uma sala climatizada, onde foram montados todos os equipamentos. Os equipamentos necessários para a pesquisa foram fornecidos pelo Laboratório de Bioengenharia e Biomecânica da Universidade Federal de Goiás.

A seleção das gestantes para este estudo foi por amostragem de conveniência. O período de coleta de dados aconteceu entre agosto e setembro de 2011. No total, 90 gestantes foram convidadas a participar do estudo. Os critérios de inclusão foram idade gestacional $-1^{\circ}$ trimestre (4-12 semanas), $2^{\circ}$ trimestre (13-28 semanas) e $3^{\circ}$ trimestre (29-42 semanas) - , faixa etária entre 18 e 35 anos, gestação única e de baixo risco, ausência de doença clínica ou obstétrica, ausência de desordens pulmonares, cardíacas, visuais, auditivas e cognitivas. Os critérios de exclusão foram: apresentar alguma intercorrência clínica ou obstétrica durante a realização do estudo, alterações musculoesqueléticas e neurofuncionais clinicamente comprovadas. O projeto foi submetido ao Comitê de Ética em pesquisa do Hospital de Urgência de Goiânia e aprovado (Protocolo n ${ }^{\circ}$ 074/09). As gestantes assinaram o termo de consentimento livre e esclarecido elaborado de acordo com a Resolução nº 196/96.

\section{Procedimentos experimentais}

Todas as gestantes que participaram do estudo preencheram um questionário semiestruturado de identificação contendo dados pessoais e um item referente ao histórico de doenças concomitantes. Do prontuário médico, foram colhidas as seguintes informações: data da última menstruação, idade gestacional, número de gestações e abortos. As medidas antropométricas (peso corporal e altura) foram coletadas com uma balança digital e um estadiômetro para a mensuração da altura $(\mathrm{em} \mathrm{cm})^{8}$.

Em seguida, a gestante foi posicionada sobre as plataformas de força, instrumento utilizado para análise cinética, posicionadas ao nível do percurso. O percurso foi montado com tablados móveis $(11 \mathrm{~cm}$ de altura e $54 / 50 \mathrm{~cm}$ de largura) que se encaixava em torno das duas plataformas de força (AMTI). Um tapete emborrachado $(40 \times 140 \times 0,003 \mathrm{~cm})$ foi posicionado para que a gestante não visualizasse as plataformas. Uma posição inicial foi padronizada para cada ensaio: 
postura ortostática com um pé sobre cada plataforma de força. A gestante ficou nessa posição até que fosse dado um comando sonoro para iniciar o passo. Foram feitas vários ensaios para cada gestante e a análise foi realizada com cinco tentativas consideradas válidas.

Os dados foram coletados pelo programa Gforce a uma frequência de $100 \mathrm{~Hz}$, e posteriormente exportados através do software BioAnalyzis para o formato texto (txt). O tratamento dos dados em formato txt foi feito em ambiente Matlab, no qual foi construída uma rotina para calcular o comportamento do COP (amplitude de oscilação e a velocidade do movimento). Os dados brutos foram suavizados por um filtro digital do tipo Butterworth de quarta ordem, com frequência de corte de $5 \mathrm{~Hz}$.

\section{Análise estatística}

Os dados foram analisados no software SPSS. Para verificar a normalidade dos dados foi utilizado o teste de Kolmogorov Smirnov. Para constatar se houve diferença entre os grupos para a variável amplitude de oscilação e velocidade aplicou-se o teste de KruskalWallis com post-hoc de Tukey. A associação entre a semana gestacional e as variáveis do COP foi realizada por meio do coeficiente de Spearman. Em todas as análises considerou-se o nível de significância de 5\%.

\section{Resultados}

Foram convidadas para o estudo, 90 gestantes, subdivididas em três grupos: grupo GPT (gestantes de primeiro trimestre), grupo GST (de segundo trimestre) e grupo GTT (de terceiro trimestre). Destas, 33 foram excluídas, sendo 4 menores de 18 anos, 6 maiores de 35, 8 com gestação de alto risco, 5 com doenças cardíacas ou pulmonares, 1 com gestação de gêmeos e 9 que não quiseram participar.

A Tabela 1 descreve as características antropométricas nos 3 trimestres de gestação das 57 gestantes que foram avaliadas.

O comportamento das variáveis do COP: amplitude de oscilação anteroposterior (COPAP), amplitude de oscilação médio-lateral (COPML), velocidade anteroposterior e velocidade médio-lateral entre os trimestres estão indicados na Tabela 2. Quando foi verificada a associação entre as variáveis do COP, não foram indicadas diferenças significativas nos três trimestres, exceto, para a variável COPML plataforma 2: $\operatorname{GPT}(0,4 \mathrm{~cm})$ e GTT $(0,2 \mathrm{~cm})$ (Tabela 2$)$.

As variáveis COPAP e COPML nas plataformas 1 e 2 tiveram seus valores reduzidos à medida que houve aumento na idade gestacional (Tabela 2). Ao se avaliar a velocidade de oscilação podemos observar que a redução mantém-se quando observamos a velocidade de deslocamento anteroposterior (VELAP) na plataforma 1 no grupo GPT $(2,8 \mathrm{~cm} / \mathrm{s})$ em relação ao grupo GTT $(2,5 \mathrm{~cm} / \mathrm{s})$ e,
Tabela 1. Características antropométricas das gestantes dos três trimestres

\begin{tabular}{lccc}
\hline Características & $\begin{array}{c}\text { GPT } \\
(\mathrm{n}=19)\end{array}$ & $\begin{array}{c}\text { GST } \\
(\mathrm{n}=19)\end{array}$ & $\begin{array}{c}\text { GTT } \\
(\mathrm{n}=19)\end{array}$ \\
\hline Idade (anos) & $29,2 \pm 4,5$ & $25,9 \pm 3,9$ & $27,1 \pm 4,6$ \\
Massa (kg) & $60,4 \pm 10,2$ & $67,8 \pm 11,4$ & $72,7 \pm 1,6^{*}$ \\
Estatura (m) & $1,6 \pm 0,1$ & $1,6 \pm 0,1$ & $1,6 \pm 0,1$ \\
\hline
\end{tabular}

Os valores estão indicados em média e desvio padrão. GPT: gestantes do primeiro trimestre; GST: gestantes do segundo trimestres; GT: gestantes do terceiro trimestre. *Diferença estatística significante em relação ao primeiro trimestre $(p=0,003)$.

Tabela 2. Variáveis do centro de pressão nos três trimestres gestacionais

\begin{tabular}{llll}
\hline Variáveis & $\begin{array}{c}\text { GPT } \\
(\mathrm{n}=19)\end{array}$ & $\begin{array}{c}\text { GST } \\
(\mathrm{n}=19)\end{array}$ & $\begin{array}{c}\text { GTT } \\
(\mathrm{n}=19)\end{array}$ \\
\hline COPAP Plał1 (cm) & $1,3 \pm 2,5$ & $0,8 \pm 0,7$ & $0,6 \pm 0,8$ \\
COPAP Plał2 (cm) & $1,1 \pm 1,5$ & $1,0 \pm 0,8$ & $0,6 \pm 0,4$ \\
COPML Plał 1 (cm) & $0,4 \pm 0,9$ & $0,5 \pm 0,4$ & $0,4 \pm 0,7$ \\
COPML. Plał2 (cm) & $0,4 \pm 0,3$ & $0,3 \pm 0,2$ & $0,2 \pm 0,2^{\star}$ \\
VELAP Plał1 (cm/s) & $2,8 \pm 3,4$ & $3,3 \pm 2,8$ & $2,5 \pm 2,0$ \\
VELAP Plał2 (cm/s) & $2,8 \pm 3,5$ & $2,1 \pm 1,1$ & $1,5 \pm 0,7$ \\
VELML Plał 1 (cm/s) & $1,5 \pm 2,5$ & $1,9 \pm 1,8$ & $1,3 \pm 1,9$ \\
VELML Plał2(cm/s) & $0,9 \pm 0,7$ & $0,7 \pm 0,5$ & $0,4 \pm 0,6$ \\
\hline
\end{tabular}

Dados apresentados como média e desvio padrão. GPT: gestantes do primeiro trimestre; GST: gestantes do segundo trimestre; GT: gestantes do terceiro trimestre; Plat 1: plataforma 1; Plat2: plataforma 2; COPAP: amplitude de oscilação do centro de pressão anteroposterior; COPML: amplitude de oscilação do centro de pressão médio-lateral; VELAP: velocidade de deslocamento anteroposterior; VELML: velocidade de deslocamento médio-lateral.

*Diferença estatisticamente significante apenas entre GPT e GTT para a variável COPML Plat2 $(p=0,003)$.

no caso VELAP plataforma 2, (GPT $(2,8 \mathrm{~cm} / \mathrm{s})$ em relação ao grupo GTT $(1,5 \mathrm{~cm} / \mathrm{s})$. No caso da velocidade de deslocamento médio-lateral (VELML), percebemos que essa diferença continua a acontecer da mesma forma se mantendo decrescente, principalmente, no grupo GPT (plataforma 1: 1,5 cm/s; plataforma 2: $0,9 \mathrm{~cm} / \mathrm{s}$ ) em relação ao GTT (plataforma 1: 1,3 cm/s; plataforma 2: $0,4 \mathrm{~cm} / \mathrm{s}$ ) para as duas plataformas. A associação entre a semana gestacional de cada trimestre e as variáveis do COP mostrou correlações muito fracas e sem significância estatística.

\section{Discussão}

Este estudo descreveu o perfil do COP e suas variáveis durante a inicialização da marcha, nos três trimestres gestacionais, bem como possíveis variações dessas variáveis no decorrer da gestação, comparando os grupos e, investigou se essas alterações poderiam contribuir para risco na estabilidade dinâmica da gestante.

Verificou-se que, no decorrer da gestação, para o processo de inicialização da marcha, houve uma redução gradativa na oscilação do COP e da velocidade dessa oscilação, sendo verificadas diferenças significativas na direção médio-lateral quando comparados os primeiro e terceiro trimestres gestacionais. $\mathrm{Na}$ gestante, o COP se move em direção ao antepé e é trazido para a base de 
suporte aumentando a pressão plantar no retropé e essa pressão é aumentada com o decorrer da gestação?.

O comportamento do COP na postura estática demonstra que essa variável tende a produzir uma maior oscilação nas direções anteroposterior e médio-lateral com o decorrer da gestação. Isso é confirmado em um estudo que avaliou o equilíbrio postural estático em gestantes frente às alterações ocorridas no corpo feminino nos três trimestres gestacionais ${ }^{10}$. Os resultados mostraram um aumento na amplitude de oscilação anteroposterior do COP nas gestantes no terceiro trimestre em relação ao primeiro e ao controle. Neste trabalho não foram encontradas diferenças significativas na amplitude de oscilação anteroposterior entre os três grupos avaliados. Este resultado poder ser justificado por se tratar de uma avaliação da inicialização da marcha e não de uma postura estática, onde ocorre uma adaptação para a manutenção do equilíbrio no processo de mudança da postura estática para o início da marcha ${ }^{11}$.

Neste estudo verificou-se que durante o processo de inicialização da marcha, no decorrer da gestação, houve uma diminuição gradativa na amplitude de oscilação do COP e da velocidade, sendo verificadas diferenças significativas na direção médio-lateral quando comparados os primeiro e terceiro trimestres gestacionais. O COP é uma medida de deslocamento dependente do centro de gravidade (CG) e expressa a localização do vetor resultante da força de reação do solo (FRS) em uma plataforma de força ${ }^{12} \mathrm{e}$, ainda, projeta o CG dentro da base de sustentação, decorrente de uma resposta neuromuscular ao balanço do CG. No caso das gestantes, fatores como ganho de peso, aumento abdominal e das mamas podem levar a uma perturbação do CG que, consequentemente, refletirá no $\mathrm{COP}^{10}$. Essas alterações podem levar a uma instabilidade no equilíbrio da gestante influenciando a biomecânica de sua postura estática e dinâmica podendo aumentar o risco de quedas ${ }^{3}$.

Quando verificadas as variáveis COPML e VELML percebeu-se que houve uma redução na oscilação dessas variáveis, quando comparados o primeiro e terceiro trimestres. Os dados sugerem que as gestantes iniciam a marcha mais vagarosamente, com maior cuidado, com redução da velocidade de deslocamento e amplitude dos passos. Essas alterações foram visualizadas na plataforma 2, onde estava posicionado o pé de apoio durante a fase de inicialização da marcha. Para minimizar os efeitos do desequilíbrio corporal, a gestante mantém os pés no chão por um maior período de tempo e mostra que esta é uma característica similar à marcha de idosos e, ainda persiste por quatro meses após o parto ${ }^{13}$.

Pode-se observar uma semelhança dos resultados obtidos neste com estudo com os indivíduos obesos onde há um menor impulso inicial e, consequentemente, a uma menor oscilação do $\mathrm{COP}^{14}$. Noutro trabalho que confirma estes achados, foi feita a avaliação da adaptação no controle estático e dinâmico em pacientes obesos e verificou-se que a maioria dos parâmetros relacionados à dinâmica das forças gravitacionais são reduzidas nesses sujeitos ${ }^{15}$.

Quando há uma situação de instabilidade postural, a resposta às forças gravitacionais fica mais lentas ${ }^{16}$. As gestantes associam menor velocidade da marcha como uma estratégia de adaptação para manter o equilíbrio e compensar alterações no CG devido ao aumento da carga ${ }^{17}$. Pode-se assim compreender que, no decorrer da gestação, a gestante tenta adaptar-se primeiro para depois iniciar sua marcha, a fim de diminuir riscos de instabilidade e, em consequência, a queda. Comparando um grupo de gestantes a um grupo de não gestantes, verificou-se que as grávidas, no decorrer da gestação, têm redução na sua habilidade de manter o equilíbrio ${ }^{18}$, o que reafirma os dados obtidos neste estudo ${ }^{19}$.

Em análise de trabalhos já descritos, nos quais se avaliou o processo de inicialização da marcha entre indivíduos jovens, idosos saudáveis e idosos com doença de Parkinson, os resultados mostraram que os idosos possuem um controle da inicialização da marcha mais lenta (padrões musculares, cinéticos e cinemáticos) quando comparados aos mais jovens ${ }^{20}$. Os indivíduos portadores de uma lesão, no caso artrose no joelho, têm suas estratégias de equilíbrio e controle de movimento mais lentas durante a inicialização da marcha, sendo assimétrica nos dois membros e desenvolvem estratégias posturais e motoras a fim de adaptar-se ao movimento ${ }^{21}$. O sistema vestibular também tem papel importante na inicialização da marcha ${ }^{22}$.

Algumas limitações foram verificadas durante a realização deste estudo. Primeiro, o número maior de gestantes é indispensável para uma melhor distribuição dos dados permitindo, assim, a análise com maior confiabilidade. Segundo, muitas gestantes não aceitaram participar do estudo, o que também dificultou a execução do mesmo. Por último, o reduzido período de coletas mostrou-se outro fator de dificuldade no acompanhamento das gestantes nos três trimestres, visto que seria importante acompanhá-las para construir um padrão biomecânico normal para a inicialização da marcha nas mesmas.

Em termos biomecânicos houve uma redução de velocidade das gestantes para realizar o início do primeiro passo, que pode ser explicado por uma adequação do corpo ao aumento do peso corporal no decorrer do período gestacional. Essas alterações na velocidade não apresentam risco à gestante, mas, sim um novo ajuste na postura para o andar independente. Essas alterações indicam a necessidade de preparar estas gestantes com o objetivo de diminuir as consequências funcionais que podem ser provocadas por essa situação de instabilidade. 
1. Mann L, Kleinpaul JF, Mota CB, Santos SG. Alterações biomecânicas durante o período gestacional: uma revisão. Motriz. 2010;16(3):730-41.

2. Benetti FA, Pegoretti C, Wittig DS, Deprá PP, Campos MH, Sabatino $\mathrm{JH}$ et al. Curvatura lombar e inclinação do tronco durante o período gestacional. Rev Ciênc Méd. 2005; 14(3):259-65.

3. Butler EE, Colón I, Druzin M, Rose J. An investigation of gait and postural balance during pregnancy. Gait Posture. 2006;24 (Suppl 2):S128-9.

4. Albino MAS, Moccellin AS, Firmento BS, Driusso P. [Gait force propulsion modifications during pregnancy: effects of changes in feet's dimensions]. Rev Bras Ginecol Obstet. 2011;33(7):164-9 Portuguese.

5. Ribeiro AP, Trombini-Souza F, Sacco ICN, Ruano R, Zugaib M, João SMA. Changes in the plantar pressure distribution during gait throughout gestation. J Am Podiatr Med Assoc. 201 1;101 (5):415-23.

6. Vinti $M$, Couillandre A, Thoumie P. Does somatosensory loss induce adaptation of the gait initiation process? Neurosci Lett. 2010;480(3):178-81.

7. McCrory JL, Chambers AJ, Daftary A, Redfern MS. Dynamic postural stability during advancing pregnancy. J Biomech. 2010;43(12):2434-9.

8. Santos MMAS, Baião MR, Barros DC, Pinto AA, Pedrosa PLM, Saunders C. Estado nutricional pré-gestacional, ganho de peso materno, condições da assistência pré-natal e desfechos perinatais adversos entre puérperas adolescentes. Rev Bras Epidemiol. 2012;15(1): 143-54.

9. Nyska M, Sofer D, Porat A, Howard CB, Levi A, Meizner I. Planter foot pressures in pregnant women. Isr J Med Sci. 1997;33(2): 139-46.

10. Ribas SI, Guirro ECO. Análise da pressão plantar e do equilíbrio postural em diferentes fases da gestação. Rev Bras Fisioter. 2007; 11 (5):391-6.

11. Lymbery JK, Gilleard W. The stance phase of walking during late pregnancy: temporospatial and ground reaction force variables. J Am Podiatr Med Assoc. 2005;95(3):247-53.
12. Melo PS, Ferreira TP, Santos-Pontelli TEG, Carneiro JAO, Carneiro AAO, Colafêmina JF. Comparação da oscilação postural estática na posição sentada entre jovens e idosos saudáveis. Rev Bras Fisioter. 2009; 13(6):549-54.

13. Carpes FP, Griebeler D, Kleinpaul JF, Mann L, Mota CB. Women able-bodied gait kinematics during and post pregnancy period. Rev Bras Biomec. 2008;9(16):33-40.

14. Patchay S, Gahéry Y. Effect of asymmetrical limb loading on early postural adjustments associated with gait initiation in young healthy adults. Gait Posture. 2003; 18(1):85-94.

15. Colné $P$, Frelut ML, Pérès $G$, Thoumie P. Postural control in obese adolescents assessed by limits of stability and gait initiation. Gait Posture. 2008;28(1):164-9.

16. Polcyn AF, Lipsitz LA, Kerrigan DC, Collins JJ. Age-related changes in the initiation of gait: degradation of central mechanisms for momentum generation. Arch Phys Med Rehabil. 1998;79(12):1582-9.

17. Santos MRS, Gil BMC, Marques AS, Vilas Boas JP, Silva JF. Comparative analysis of the ground reaction forces, during the support phase, in a group of pregnant women on their $3^{\text {rd }}$ trimester of pregnancy and in a group pf not pregnant women. Fisioter Mov. 2008;21(1):95-103.

18. Kleinpaul JF, Mann L, Teixeira CS, Lopes LFD, Konopka CK, Mota CB. Gestação: equilíbrio corporal, dor lombar e quedas. Rev Bras Biomec. 2009;10(18):14-21.

19. Nagai $M$, Isida $M$, Saitoh J, Hirata Y, Natori H, Wada M. Characteristics of the control of standing posture during pregnancy. Neurosci Lett. 2009;462(2):130-4.

20. Halliday SE, Winter DA, Frank JS, Patla AE, Prince F. The initiation of gait in young, elderly, and Parkinson's disease subjects. Gait Posture. 1998;8(1):8-14.

21. Viton JM, Timsit M, Mesure S, Massion J, Franceschi JP, Delarque A. Asymmetry of gait initiation in patients with unilateral knee arthritis. Arch Phys Med Rehabil. 2000;81 (2):194-200.

22. Sasaki O, Asawa S, Katsuno S, Usami S, Taguchi K. Gait initiation in bilateral vestibular loss. Auris Nasus Larynx. 2001 ;28(4):295-9. 